\title{
High-Output Heart Failure in Mitochondrial Myopathy: A Fulminant Form with Severe Lactic Acidosis and Rhabdomyolysis
}

\author{
Yasuo Hirose, Tsutomu MiIdA*, Kazukiyo YoshidA**, Hideki KimurA**, \\ Hirotaka OdA*, Tetsuro ToedA* and Norio HigumA*
}

\begin{abstract}
A 17-year-old male with mitochondrial myopathy was transferred to our hospital because of respiratory distress and a rapidly deteriorating level of consciousness. He had a markedly increased cardiac output with low systemic vascular resistance and decreased arteriovenous oxygen difference. Severe lactic acidosis, rhabdomyolysis and acute renal failure were also present. He was treated in the intensive care unit with continuous hemofiltration and hemodialysis. This case demonstrates that cardiac failure in mitochondrial encephalomyopathy may be caused by myocardial abnormalities as well as by reduced systemic vascular resistance secondary to disturbed oxidative metabolism.
\end{abstract}

(Internal Medicine 32: 798-801, 1993)

Key words: NADH-coenzyme Q reductase (complex I) deficiency, acute renal failure, blood purification, shoshin beriberi.

\section{Introduction}

The mitochondrial encephalomyopathies are a heterogeneous group of syndromes involving the muscles and the central nervous system and featuring structural abnormalities of the mitochondria (1). These disorders are often complicated by lactic acidosis due to mitochondrial dysfunction. Secondary cardiomyopathy and heart block in Kearns-Sayre syndrome are widely recognized cardiac manifestations of these conditions (2), but the occurrence of high-output heart failure appears to be rare. We present a successfully treated patient with mitochondrial myopathy complicated by life-threatening lactic acidosis, rhabdomyolysis, acute renal failure and severe high-output heart failure.

\section{Case Report}

The patient was a high school student, whose birth and early development were reported to be normal except that he suffered from excessive fatigue. Two maternal cousins had been diagnosed as having mitochondrial myopathy, and biochemical studies on their skeletal muscle mitochondria revealed defective activity of NADH-coenzyme Q reductase (complex I) in the respiratory chain. This family has been already described elsewhere (3-5). At the age of 15 years, his muscle biopsy showed abnormal mitochondria and ragged-red fibers, but mitochondrial respiratory chain enzyme defects were not documented. A T-to-C transition mutation at nucleotide position 3,250 was pointed out in his mitochondrial tDNA ${ }^{\text {Leu(UUR) }}$ gene (4). Ergometric aerobic exercise testing showed abnormally high serum lactate and pyruvate levels (5). At the age of 16 years, the patient experienced two episodes of muscle weakness, fatigue, moderate elevation of the serum creatine kinase (CK) level and metabolic acidosis, and each episode subsided in a few days in hospital. A diagnosis of mitochondrial myopathy was made and he was started on medication with 1-carnitine, coenzyme Q, and dichloroacetate (6). [This medication has been continued throughout his clinical course and up to the present time.] At the age of 17 years, he was admitted to another hospital with a 2-week history of progressive muscle weakness, easy fatigability and low grade fever. His level of consciousness and respiratory function showed rapid deterioration and he was transferred to our institution on the next day. On arrival in our emergency room, he was comatose (Glasgow Coma Scale: 3), the pulse rate was 100/min and regular, the blood pressure was $70 \mathrm{mmHg}$ by palpation, and the respiration rate was $40 / \mathrm{min}$. Laboratory data on admission is presented in Table 1. Arterial blood gas analysis while he was receiving oxygen $(5 \mathrm{l} / \mathrm{min})$

From the Critical Care Medical Center, *the Department of Cardiology and **the Department of Nephrology, Niigata City General Hospital, Niigata Received for publication April 14, 1993; Accepted for publication October 12, 1993

Reprint requests should be addressed to Dr. Yasuo Hirose, Critical Care Medical Center, Niigata City General Hospital, 2-6-1 Shichikuyama, Niigata 950 
Table 1. Laboratory Data on Admission

\begin{tabular}{lrlr}
\hline $\mathrm{pH}$ & 6.846 & $\mathrm{Na}$ & $134 \mathrm{mEq} / \mathrm{l}$ \\
$\mathrm{Pco}_{2}$ & $50.0 \mathrm{mmHg}$ & $\mathrm{K}$ & $3.8 \mathrm{mEq} / \mathrm{l}$ \\
$\mathrm{Po}_{2}$ & $173.6 \mathrm{mmHg}$ & $\mathrm{C} 1$ & $100 \mathrm{mEq} / 1$ \\
$\mathrm{HCO}_{3}$ & $8.6 \mathrm{mEq} / 1$ & $\mathrm{Ca}$ & $9.7 \mathrm{mg} / \mathrm{dl}$ \\
$\mathrm{BE}$ & $-27.0 \mathrm{mEq} / \mathrm{l}$ & $\mathrm{P}$ & $14.8 \mathrm{mg} / \mathrm{dl}$ \\
$\mathrm{So}_{2}$ & $97.7 \%$ & $\mathrm{TP}$ & $6.6 \mathrm{~g} / \mathrm{dl}$ \\
& $\left(\mathrm{O}_{2} 5 \mathrm{l} / \mathrm{min}\right)$ & $\mathrm{Alb}$ & $4.7 \mathrm{~g} / \mathrm{dl}$ \\
& & $\mathrm{BUN}$ & $20 \mathrm{mg} / \mathrm{dl}$ \\
$\mathrm{WBC}$ & $14,100 / \mu \mathrm{l}$ & $\mathrm{Cre}$ & $1.3 \mathrm{mg} / \mathrm{dl}$ \\
$\mathrm{RBC}$ & $495 \times 10^{4} / \mu \mathrm{l}$ & $\mathrm{GOT}$ & $134 \mathrm{IU} / 1$ \\
$\mathrm{Hb}$ & $15.0 \mathrm{~g} / \mathrm{dl}$ & $\mathrm{GPT}$ & $29 \mathrm{IU} / 1$ \\
$\mathrm{Ht}$ & $46.1 \%$ & $\mathrm{LDH}$ & $1,623 \mathrm{IU} / 1$ \\
$\mathrm{Plt}$ & $30.4 \times 10^{4} / \mu \mathrm{l}$ & $\mathrm{ALP}$ & $118 \mathrm{IU} / 1$ \\
& & $\mathrm{CPK}$ & $1,446 \mathrm{IU} / 1$ \\
& & $\mathrm{CPK}-\mathrm{MB}$ & $17 \%$ \\
Lactate & $191.2 \mathrm{mg} / \mathrm{dl}$ & $\mathrm{UA}$ & $14.8 \mathrm{mg} / \mathrm{dl}$ \\
& & $\mathrm{T} . \mathrm{Bil}$ & $0.5 \mathrm{mg} / \mathrm{dl}$ \\
$\mathrm{CRP}$ & $(-)$ & $\mathrm{T} . \mathrm{CHO}$ & $154 \mathrm{mg} / \mathrm{dl}$ \\
\hline
\end{tabular}

delivered by a face mask showed severe metabolic acidosis: pH 6.85; $\mathrm{PaCO}_{2} 50$ torr; $\mathrm{PaO}_{2} 174$ torr; serum bicarbonate, 8.6 $\mathrm{mmol} / \mathrm{l}$; and base deficit, $27 \mathrm{mmol} / \mathrm{l}$. The white blood count (WBC) was elevated at $14,100 / \mathrm{mm}^{3}$. In addition, he had elevated serum levels of glutamic oxaloacetic transaminase (GOT, $134 \mathrm{U} / \mathrm{l})$, lactate dehydrogenase (LDH, 1,623 U/l), CK (1,446 $\mathrm{U} / \mathrm{l})$ and lactate $(191.1 \mathrm{mg} / \mathrm{dl})$. Soon after he was admitted to the intensive care unit, cardiopulmonary arrest occurred and resuscitation was begun. He was resuscitated immediately, but hypotension persisted despite large doses of dopamine and norepinephrine. The chest X-ray film showed cardiomegaly and bilateral pulmonary congestion (Fig. 1), and the electrocar-

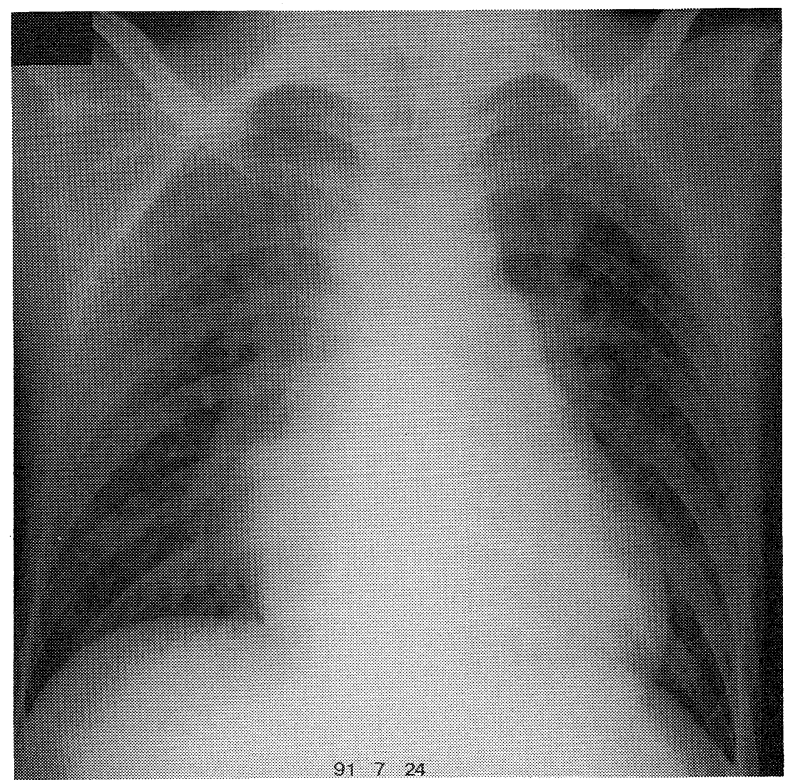

Fig. 1. Chest X-ray findings on admission showed cardiomegaly and bilateral pulmonary congestion.

diogram demonstrated sinus tachycardia and nonspecific ST segment changes. Echocardiography showed normal left ventricular contraction. A thermodilution pulmonary artery catheter was inserted and the hemodynamic data obtained indicated severe high-output heart failure. The cardiac output was 20.8 1/ min, the pulmonary artery pressure was $40 / 30 \mathrm{mmHg}$, the pulmonary artery wedge pressure was $21 \mathrm{mmHg}$, and the mixed

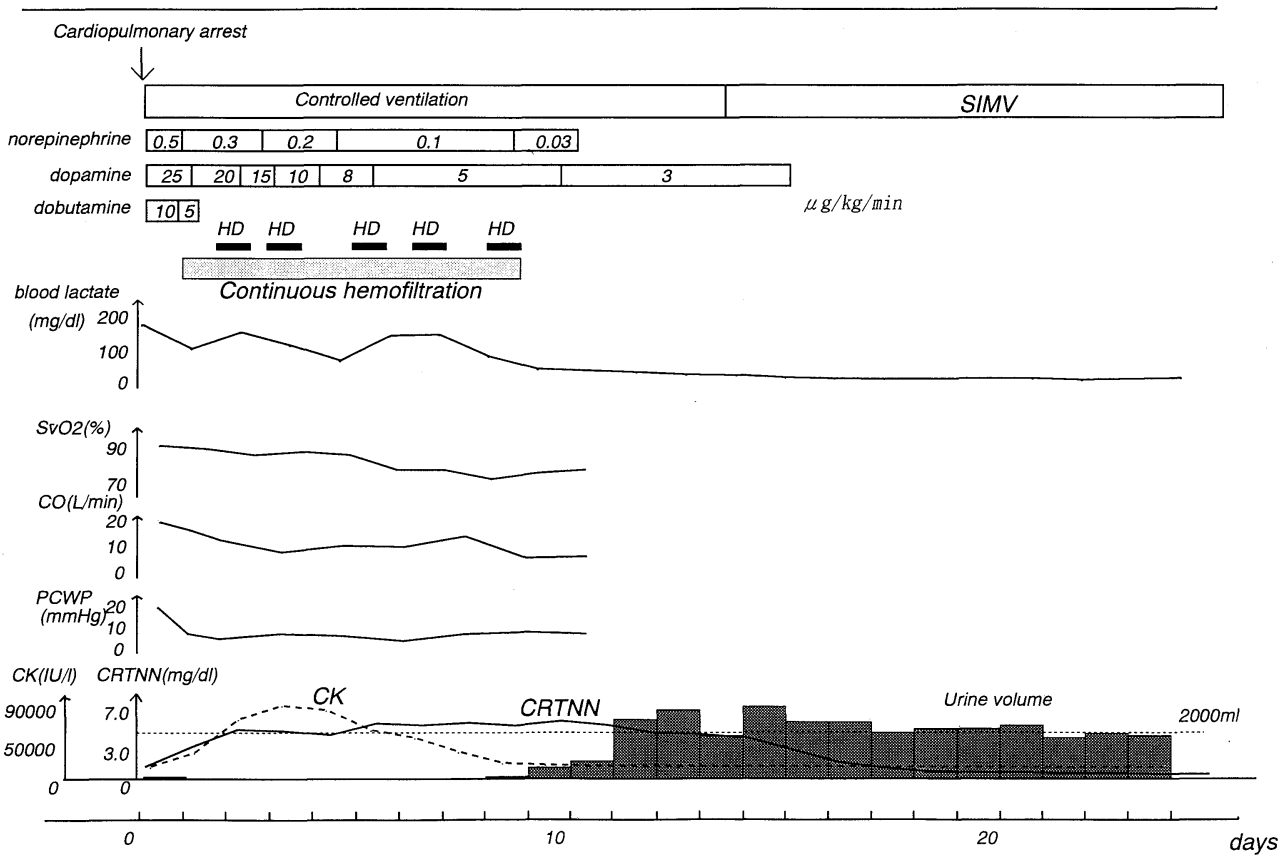

Fig. 2. Clinical course in the intensive care unit. HD: hemodialysis, SvO2: mixed venous oxygen saturation, CO: cardiac output, PCWP: pulmonary capillary wedge pressure, CK: creatine kinase, CRTNN: Creatinine. 
venous oxygen saturation was $93 \%$. Severe metabolic acidosis persisted despite the administration of high doses of sodium bicarbonate. The serum $\mathrm{CK}$ level rose rapidly and peaked at $92,125 \mathrm{U} / \mathrm{l}$ on the 4 th hospital day. Anuric acute renal failure developed immediately after admission, and the serum creatinine level peaked at $7.0 \mathrm{mg} / \mathrm{dl}$ on the 8 th hospital day. Continuous hemofiltration and intermittent hemodialysis were performed from hospital days 2 to 8 . The mixed venous oxygen saturation and the high cardiac output state were gradually normalized and he was weaned from the infusion of norepinephrine and dopamine. His azotemia was normalized following the improvement of rhabdomyolysis and urine production was detected by day 9 . His conscious state and mental function were recovered completely, but he showed severe muscular atrophy and tetraplegia. Respiration remained very weak and weaning from the respirator was quite difficult. The weakness was prolonged and he could only be successfully weaned from assisted respiration after 3 months. Echocardiography, electrocardiography and thallium-201 myocardial scintigraphy at rest were within normal limits. After rehabilitation for 10 months there was a gradual return of muscle power to his upper extremities, but the weakness of lower extremities persisted. He is now able to sit in a wheelchair by himself.

\section{Discussion}

Recent biochemical investigations of muscle mitochondria in mitochondrial encephalomyopathy have revealed certain specific metabolic defects. Deficiency of NADH-coenzyme Q reductase (complex I) in the respiratory chain was first described by Morgan-Hughes et al (7), and has been classified into 4 clinical types: the fatal infantile type, the myopathic type, the encephalomyopathic type without stroke-like episodes, and mitochondrial myopathy, encephalopathy, lactic acidosis, and stroke-like episodes (MELAS syndrome) (8). Our patient underwent pathological and biochemical analyses of skeletal muscles at the age of 15 years; histological examination showed characteristic abnormalities of these disorders and a point mutation in the mitochondrial tRNA was detected, although mitochondrial enzyme defects were not documented $(4,5)$. The patient was strongly suspected of having an NADH-coenzyme $\mathrm{Q}$ reductase deficiency and was clinically classified as the myopathic type on account of his maternal family history (3-5).

Mitochondrial encephalomyopathies are often complicated by cardiomyopathy (2,9-12), and characteristic mitochondrial abnormalities similar to those seen in skeletal muscle have been described in the myocardium (10-12). Nishizawa et al reported a patient with the clinical features of MELAS syndrome and cardiomyopathy who demonstrated a deficiency of NADHcoenzyme Q reductase (complex I) activity in the myocardium (10). Congestive heart failure in patients with these disorders is generally considered to be caused by impairment of myocardial contraction. In our patient, however, the cardiac output was markedly increased and the arteriovenous oxygen difference was decreased, in addition to severe lactic acidosis and hypotension refractory to inotropic agents.
We noted a close analogy of this patient's hemodynamic data to those obtained in shoshin beriberi. Shoshin beriberi is a fulminating form of beriberi heart disease caused by thiamine deficiency, and is characterized by hypotension, tachycardia, and lactic acidosis. Patients with shoshin beriberi have an increased cardiac output and a reduction of the arterial-mixed venous oxygen difference and the systemic vascular resistance. The right and left ventricular filling pressures and circulating blood volume are also elevated (13). Thiamine is required as a cofactor for energy production at two important decarboxylation steps in the Krebs cycle and the respiratory chain, so thiamine deficiency results in dysfunction of oxidative metabolism with a consequent accumulation of pyruvate and lactate.

With regard to the disturbance of oxidative metabolism and the accumulation of pyruvate and lactate, our patient had very similar features to those of beriberi. In mitochondrial myopathies, disturbance of enzymic reactions causes the overproduction of lactate. In our patient, peripheral vasodilatation resulted therefrom and a compensatory increase of the cardiac output occurred. Echocardiography showed that myocardial contractility was preserved despite the presence of circulatory failure, suggesting that this was mainly due to low systemic vascular resistance rather than to myocardial abnormalities. Several patients of congestive heart failure associated with mitochondrial encephalomyopathies have been reported (9-12, $14,15)$, although the hemodynamic data were not provided. Only Mastaglia et al reported a 23-year-old man with mitochondrial myopathy and high-output heart failure and suggested a resemblance of the condition to beriberi (15). The present patient's blood thiamine level and transketolase activity in the erythrocytes were not examined during the last episode. He had eaten a balanced diet until that time and it is virtually unlikely that he was in a state of thiamine deficiency.

Our patient required intensive care because of severe lactic acidosis, rhabdomyolysis and acute renal failure. We used continuous hemofiltration to remove fluid overload, thereby decreasing pulmonary edema, and to provide parenteral nutrition. We performed hemodialysis to treat the lactic acidosis and the azotemia. Continuous blood purification is a useful method to manage anuric patients with circulatory instability and hypotension (16). Since no studies documenting a fulminant form of mitochondrial encephalomyopathy, as seen in the presented case, have appeared so far to date in the literature, the optimum treatment for this pathogenic condition remains to be established.

His muscle weakness showed a tendency to improve with long-term rehabilitation. After three months he was weaned from the respirator and after 10 months he could sit in a wheelchair by himself. It is generally thought that muscle weakness in this disease as well as in related pathogenic conditions is irreversible and irremediable. Since, however, more improvement of muscle power may be anticipated in acutely progressive cases (as in the present case), than in chronic cases, careful and persevering rehabilitation should be attempted.

In conclusion, high-output heart failure developed in asso- 


\section{Heart Failure in Mitochondrial Myopathy}

ciation with severe lactic acidosis and rhabdomyolysis in the present patient. Cardiac failure in mitochondrial encephalomyopathy may be caused by myocardial abnormalities as well as by reduced systemic vascular resistance secondary to dysfunction of oxidative metabolism.

\section{References}

1) Shapira Y, Harel S, Russell A. Mitochondrial encephalomyopathies. A group of neuromuscular disorders with defects in oxidative metabolism. Isr J Med 13: 161, 1977.

2) Tanaka H. Secondary cardiomyopathy associated with myopathy. Nippon Naika Gakkai Zasshi 80: 220, 1991 (in Japanese).

3) Tohyama J, Torigoe K, Sato S, et al. A case of complex I deficiency with episodic respiratory distress. No to Hattatsu 22: 369, 1990 (in Japanese).

4) Goto Y, Tojo M, Tohyama J, Horai S, Nonaka I. A novel point mutation in the mitochondrial tRNA ${ }^{\text {Leu(UUR) }}$ gene in a family with mitochondrial myopathy. Ann Neurol 31: 672, 1992.

5) Tojo M, Ogawa N, Takeuchi M, et al. Ergometric and pathologic study of a family with complex I deficiency. No to Hattatsu 24: 20, 1992 (in Japanese).

6) Przyrembel H. Therapy of mitochondrial disorders. J Inherit Metab Dis 10: $129,1987$.

7) Morgan-Hughes JA, Darveniza P, Landon DN, Land JM, Clark JB. A mitochondrial myopathy with a deficiency of respiratory chain NADHCoQ reductase activity. J Neurol Sci 43: 27, 1979.
8) Ichiki T, Tanaka M, Nishikimi M, et al. Deficiency of subunits of complex I and mitochondrial encephalomyopathy. Ann Neurol 23: 287, 1988.

9) Sengers RCA, Stadhouders AM, Lakwijk-Vondrovicova E, Kubat K, Ruitenbeek W. Hypertrophic cardiomyopathy associated with a mitochondrial myopathy of voluntary muscles and congenital cataract. $\mathrm{Br}$ Heart J 54: 543, 1985.

10) Nishizawa $M$, Tanaka $K$, Shinozawa $K$, et al. A mitochondrial encephalomyopathy with cardiomyopathy. A case revealing a defect of complex I in the respiratory chain. J Neuro Sci 78: 189, 1987.

11) Nemoto $T$, Satoh $W$, Harada $K$, et al. Cardiac involvement in four cases of MELAS (mitochondrial myopathy, encephalopathy, lactic acidosis and stroke-like episodes). Nippon Shounika Gakkai Zasshi 93: 1416, 1989 (in Japanese).

12) Channer KS, Channer JL, Campbell MJ, Russell Rees J. Cardiomyopathy in the Kearns-Sayre syndrome. Br Heart J 59: 486, 1988.

13) Akbarian M, Yankopolous NA, Abelmann WH. Hemodynamic studies in beriberi heart disease. Am J Med 41: 197, 1966.

14) Oldfors A, Tulinius M, Holmes E, Kristiansson B, Eriksson BO. Mitochondrial encephalomyopathy. A variant with heart failure and liver steatosis. Acta Neuropathol 74: 287, 1987.

15) MastagliaFL, Thompson PL, Papadimitrious JM. Mitochondrial myopathy with cardiomyopathy, lactic acidosis and response to prednisolone and thiamine. Aust NZ J Med 10: 660, 1980.

16) Hirasawa $H$, Sugai $T$, Ohtake $Y$, et al. Continuous hemofiltration and hemodiafiltration in the management of multiple organ failure. Contrib Nephrol 93: 42, 1991. 